\title{
In vitro evaluation of QR-313; an antisense oligonucleotide designed to skip exon 73 from the COL7A1 mRNA
}

L van Wissen ${ }^{1}$, M Schuijt ${ }^{1}$, M Potman 1 , S Mahakena ${ }^{1}$, G Platenburg ${ }^{1}$, N Nguyen ${ }^{2}$, KJ Shridhar $^{2}$, MP Marinkovich ${ }^{2}$, T Ritsema ${ }^{1}$, EM Haisma ${ }^{1}$ ProQR Therapeutics N.V. Leiden, The Netherlands|'2Stanford Junior University, California, USA

\section{Introduction}

Recessive dystrophic epidermolysis bullosa (RDEB) is a genetic blistering disorder, caused by mutations in the COL7A1 gene encoding for type VII collagen (C7) protein. These mutations result amongst other complications in fragility of the skin and mucosal membranes. RNA exon-skipping is the modulation of splicing of a pre-mRNA in order to prevent inclusion of a targeted exon into the mRNA. Many RDEB patients harbour a mutation in exon 73 of the COL7A1 gene. We aimed to identify an antisense oligonucleotide (AON) that would exclude the in-frame exon 73 from the COL7A1 mRNA (figure 1). Exon skipping in C7 by the use of AONs has been shown for exon 105, 13, 73 and 80. For C7 lacking exon 13 and 105 has been demonstrated that the protein folding, adhesion and migration was recently published that the slightly shorter collagen type VII protein produced by patient cells after skipping of exon 73 is able to be produced be incorporated at the dermal-epidermal junction and form anchoring fibrils (Turczynski et al., 2016). After initial screening QR-313, a single stranded fully phosphorothioated and 2'O-methylated AON, composed of 21 bases was selected. This sequence is optimized for length, sequence, lack of immunogenicity and optimal manufacturability.

\section{Aims}

In this study we wanted to assess exon skip efficacy in multiple cell types (including (RDEB) fibroblasts and HaCat cells). In addition we wanted to test the exon skip potential of QR-313 when applied topically in a hydrogel formulation onto wounded human skin equivalents (HSEs).

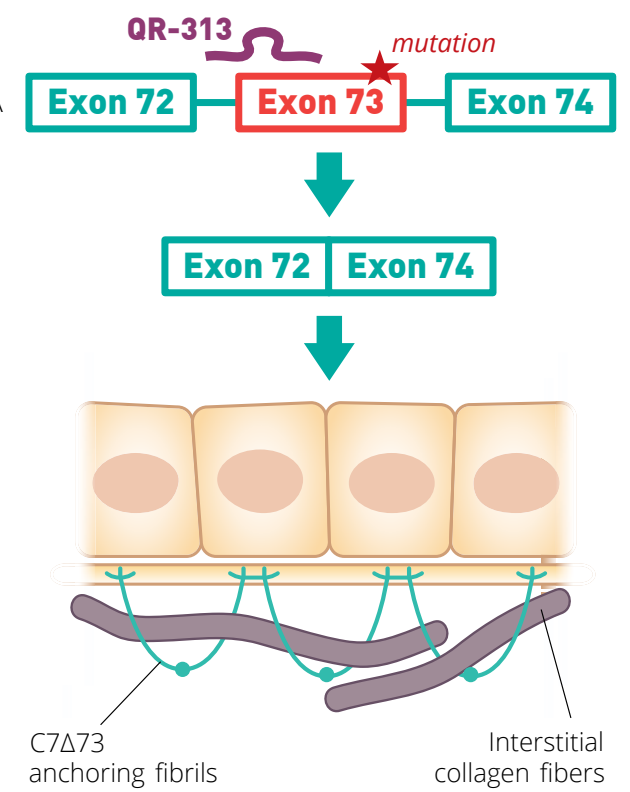

gure 1. Mode of ction of QR-313. 3 inclusion in the C7D73
anchoring fibrils Interstitial to create a slightly

Material and Methods

\section{In vitro transfection of cell cultures}

Exon skip efficacy of QR-313 was assessed in human primary fibroblasts (HPF), RDEB fibroblasts harbouring a mutation in exon 73, and (mutated) HaCats. Cells were transfected with 3.12- 200 nM QR-313 with polyethylenimine (maxPEI) for different time points. After this, RNA was isolated and CDNA produced. Products were analysed using polymerase chain reaction (PCR) for the exon 73 region, PCR products were visualized via Bioanalyser and semi-quantification was performed on the WT C7 efficacy was calculated with the formula described below:

\section{Exon 73 skip \% $=\frac{\text { Exon } 73 \text { skipped PCR product (nmol/L) }}{\text { Total PCR products }(n m o / L)} \times 100 \%$}

\section{Immunofluorescent staining of $\mathrm{C7}$}

WT fibroblasts or RDEB fibroblasts were plated in $\mu$-Plate 96 well plates and subsequently transfected with QR-313 as described above. 72 hours after transfection, cells were fixed with $4 \%$ formaldehyde, blocked and stained with a polyclonal Anti-Collagen VII antibody. Nuclei were stained with

\section{Exon skip measurements in human skin equivalents}

taining fibroblasts in a collagen matrix and an epidermal layer containing keratinocytes were created. The HSEs were cultured on filters (size $x$ by $x$ ) at the air-liquid interface for 14 days to induce differentiation of the epidermis and form a stratum corneum. After culturing, part of the epidermis was removed to mimiq the blister-wound phenotype of RDEB. Subsequenly, QR-313 formulated in a hydrogel was applied onto the wounded area. 48 hours after incubation the exon 73 region in COLIA1.

\section{Results}

1. Efficient exon skipping by QR-313 in wild type and RDEB fibroblasts

Exon 73 skip efficicacy of QR-313 in RDEB fibroblasts (PF) and wild type fibroblasts (HPF) was assessed by PCR. First dose response of $3.1 \mathrm{nM}$ to skip efficacy measured by PCR of $74 \%$ in HPF and 86\% in PF, this exon exclusion efficacy is further elevated to a median exon skip of $82 \%$ and $87 \%$ respectively when the cells were treated with higher concentrations of $\mathrm{QR}$ 313 (figure 2A). After 48 hours the exon 73 exclusion efficacy is further elevated to $100 \%$ for all tested concentrations in PF compared to $84 \%$ exon exclusion in HPEs treated with $200 \mathrm{nM}$ QR-313 (figure 2B). Bioanalyser data of one representative experiment in $\mathrm{PF}$ is given in figure $2 \mathrm{C}$

\section{Results (continued)}
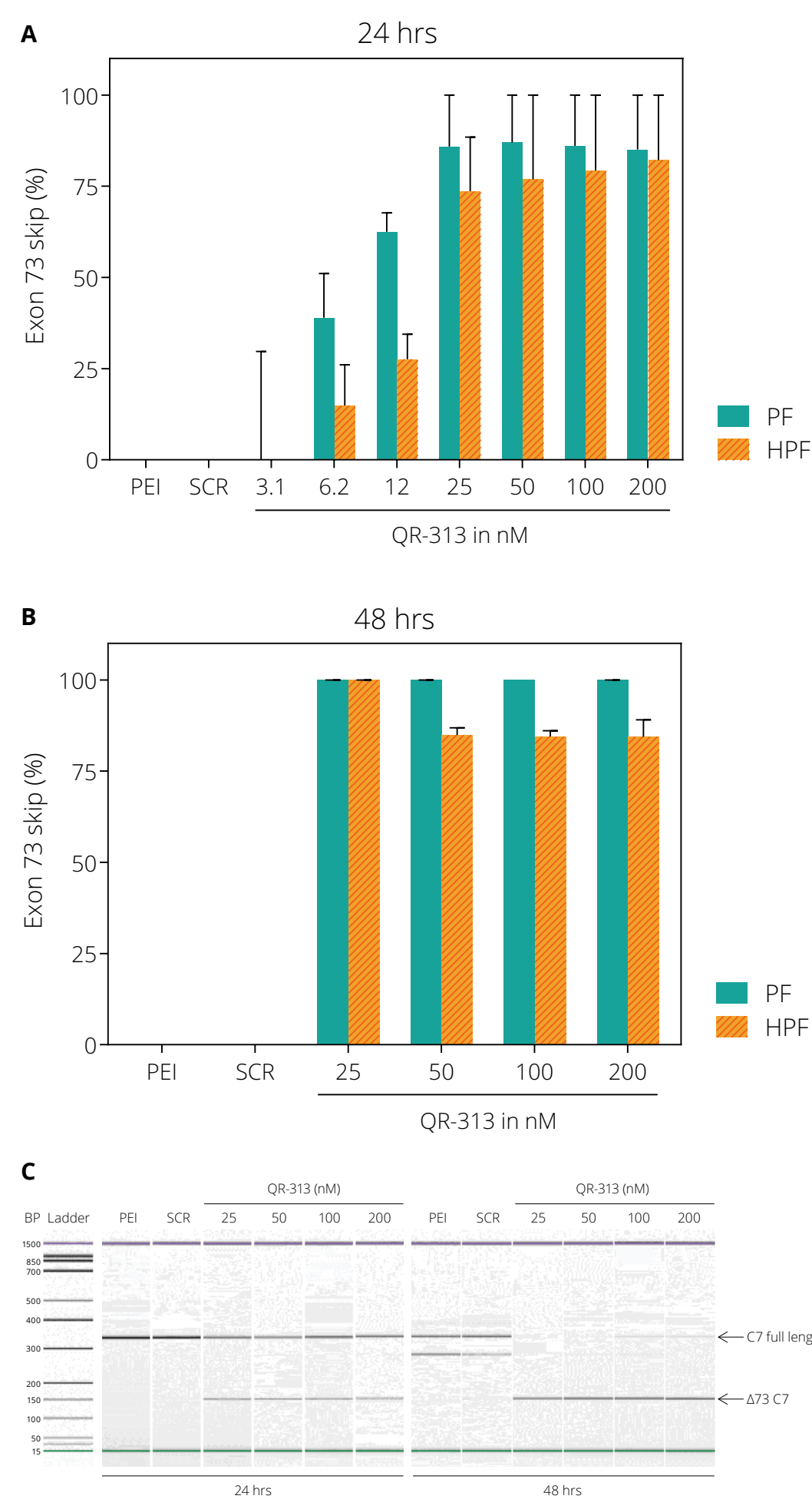

Figure 2. Exon 73 exclusion efficacy of QR-313 in PF and HPF measured by PCR. PF and HPF were transfected for (A) 24 hours or (B) 48 hours with different concentrations of QR-313 or $100 \mathrm{nM}$ or scrambled QR-313 (SCR). Exon exclusion efficacy in percentage as measured by Bioanalyzer is given. Median and range of 3 independent experiments are shown. PF RDEB fibroblasts, HPF: wild type fibroblasts. (C) Bioanalyzer results of the PF showing almost 100\% absence of the C7 wt product 48 hours after without exon $73(\Delta 73$ ( 17$)$

2. Efficient exon skipping of QR-313 in a RDEB cell-line

Using CRIPR-Cas a RDEB HaCat cell line was created by introducing a homozygous single $\mathrm{G}$ deletion in exon 73 of COL7A1, this HaCat cell-line was named HaCat Papilio ex73. Next, in both wild type HaCat cells and HaCat Papilio ex73 we assessed the exon skip efficacy of QR-313 24 hours after transfection (dose range of 3,125-100 nM QR-313). Results demonstrate that QR-313 efficiently excluded exon 73 from the COL7A1 mRNA in both HaCat and the $\mathrm{HaCat}$ Papilio ex73 cell lines, with skip efficiencies of $>50 \%$ when (figure $3 \mathrm{~A}$ ) Bionsfected with $25 \mathrm{nM}$ or higher concentrations of QR-313 figure $3 B$.

A

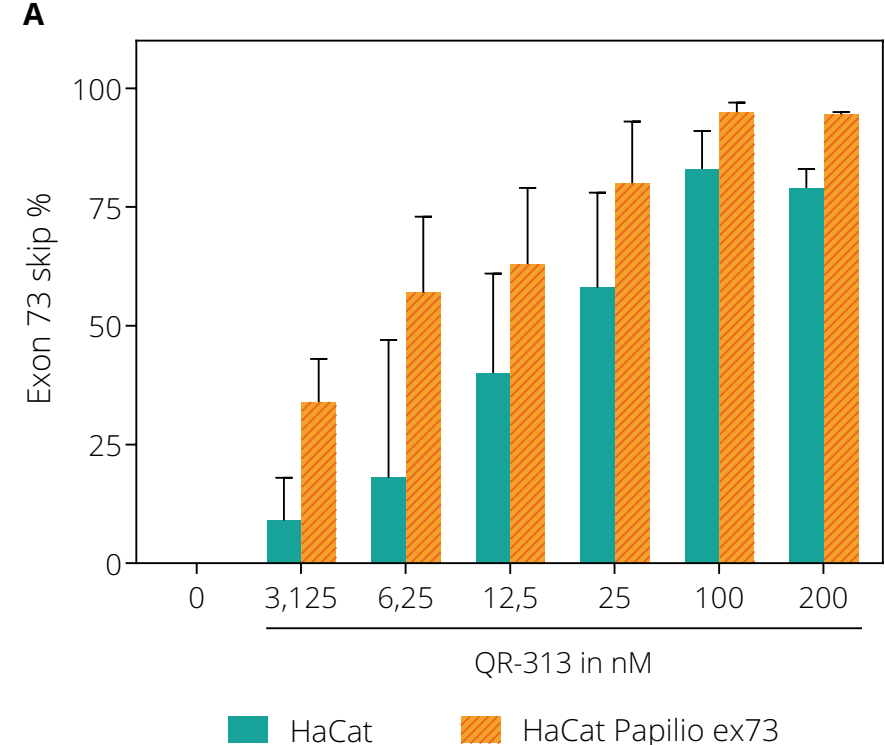

B

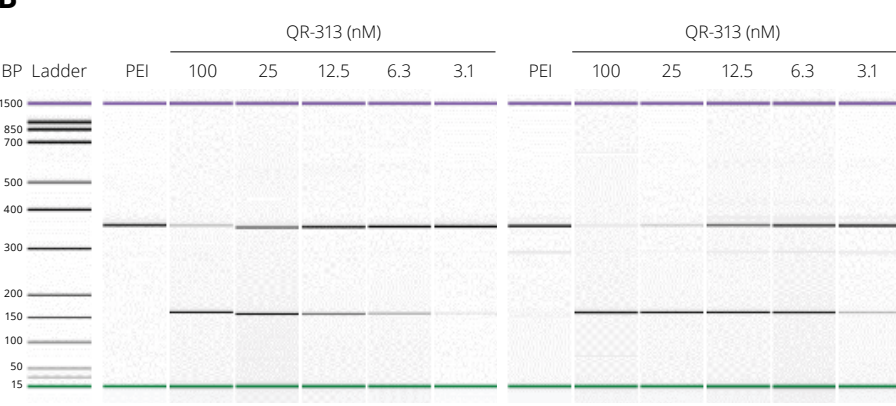

Figure 3. Dose-range of QR-313 in HaCat cells and HaCats Papilio ex 73. (A) Calculation of exon 73 skip efficacy in \%. Represeanted is median and range (B) Bioanalyser results. All cells we transfected for 24 hours and concentrations of QR-313 mentioned are in nM.
3. C7 protein expression in RDEB fibroblasts after transfection with QR-313

To assess C7 protein expression in PFs and HPFs cells were stained 72 hours after transfection with maxPEl only (Pei), $100 \mathrm{nM}$ QR-313 or $100 \mathrm{nM}$ was directed against the $\mathrm{NC1}$ domin of the C7 protein to measure both full length $C 7$ and $C 7$ without exon 73 . HPF cells show normal C7 expression with no visible difference if transfected with QR-313 or scrambled QR-313 (figure 4). The PF cells hardly express any protein when transfection with QR-313 the C7 signal in these cells is comparable to that of healthy HPF (figure 4 , second column). Note that a very low C7 staining is observed in the PFs when treated with in the maxPEl or scrambled QR-313, this could be due to presence of residual NC1 domain in these cells.
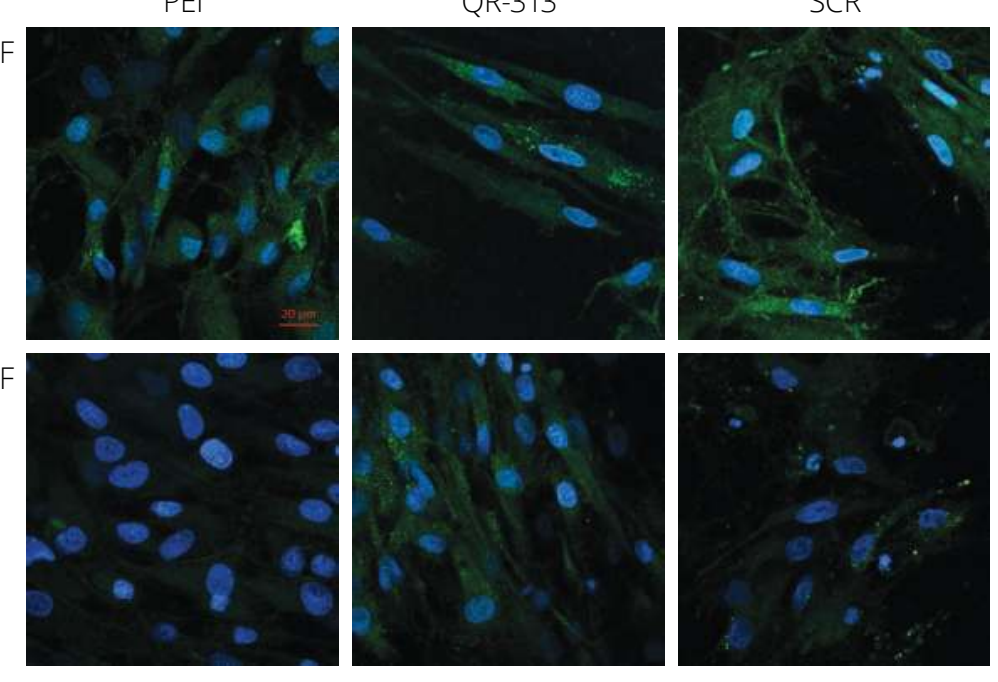

Figure 4. C7 protein expression in HPF and PF after treatment with maxPEI (PEI) only, 100 nM QR-313 or scrambled QR-313 (SCR) for 72 hours. C7 domain of C7. Nuclei are depicted in blue C7 protein in green. Scale bar is $20 \mu \mathrm{m}$.

4. Exon 73 skip demonstrated in human skin equivalents after topical treatment with QR-313 To assess the efficacy of QR-313 when applied onto HSEs, part of the epidermis was removed to mimic wounded DEB skin before QR-313 was topically applied in a hydrogel formulation. Wounding was necessary since we showed that AONs will not penetrate intact skin (data not shown). Either 10, 100 or $1000 \mu \mathrm{g}$ of QR-313 in $200 \mathrm{mg}$ of hydrogel was applied to a wound of $0.8 \mathrm{~cm}$ by $2.2 \mathrm{~cm} .24$ or 48 hours after application the dermis and epidermis were analyzed separately for exon 73 skip. We observed demonstrated exon skip after 48 hours (figure 5 .
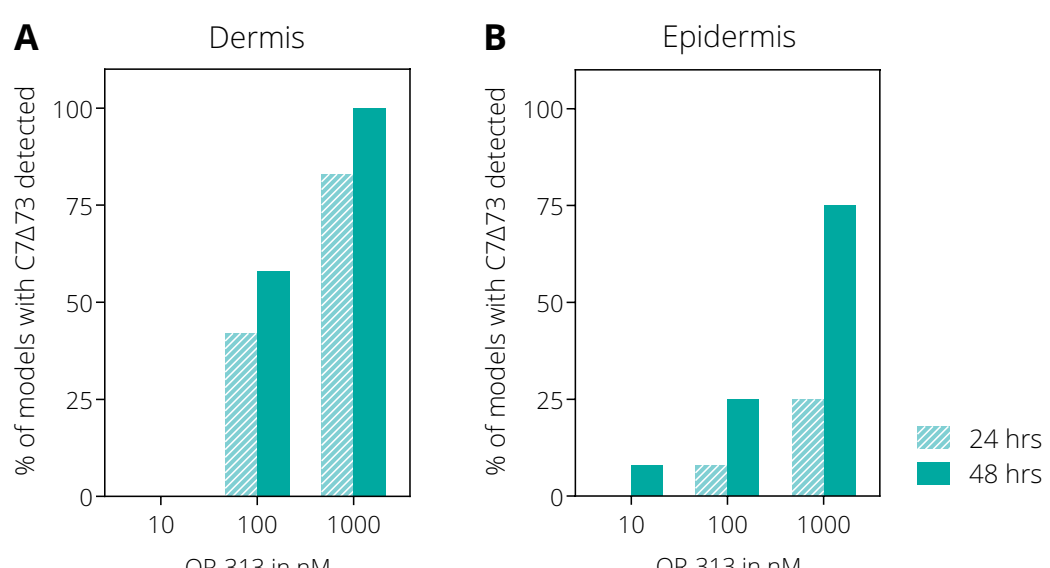

Figure 5. Percentage of HSEs showing exon skip after 24 or 48 hour treatment with QR-313 formulated into a hydrogel. Dermis (A) and Epidermis (B) were analysed separately demonstrating higher exclusion efficacy in the dermis. Results are given as the mean of 2 donors. 6 replicates per donor where performed.

\section{Conclusion}

QR-313 is very efficient in the exclusion of exon 73 from the COL7A1 mRNA in fibroblasts of an RDEB patient with a mutation in exon 73 and a HaCat cell line harboring a homozygous single $\mathrm{G}$ deletion in exon 73 . In addition, transfection with QR-313 leads to an increase in C7 protein expression in the RDEB patient fibroblasts. Moreover, hydrogel-formulated QR-313 is active in inducing exon 73 skip in COL7A1 in wounded HSEs, which mimicks the situation in patient skin.

Literature Hovnanian, A. (2016). Targeted Exon Skipping Restores Type VII Collagen Expression and Anchoring Fibril Formation in an In Vivo RDEB Model. Journa of Investigative Dermatology, 136(12), 2387-2395. http://doi.org/10.1016/j. jid.2016.07.029 prospect for dystrophic epidermolysis bullosa therapy. Molecular Therapy accepted article preview online 09 May 2016; doi:10.1038/mt.2016.92 